\title{
Applied Model-Based Analysis and Synthesis for the Dynamics, Guidance, and Control of an Autonomous Undersea Vehicle
}

\author{
Kangsoo Kim ${ }^{1}$ and Tamaki Ura ${ }^{2}$ \\ ${ }^{1}$ NTT Communication Science Laboratories, Nippon Telegraph and Telephone Corporation, \\ 3-1 Morinosato Wakamiya, Atsugi, Kanagawa 243-0198, Japan \\ 2 Institute of Industrial Science, The University of Tokyo, 4-6-1 Komaba, Meguro, Tokyo 153-8505, Japan \\ Correspondence should be addressed to Kangsoo Kim, kskim@idea.brl.ntt.co.jp
}

Received 16 August 2009; Revised 13 November 2009; Accepted 22 December 2009

Academic Editor: José Balthazar

Copyright (C) 2010 K. Kim and T. Ura. This is an open access article distributed under the Creative Commons Attribution License, which permits unrestricted use, distribution, and reproduction in any medium, provided the original work is properly cited.

\begin{abstract}
Model-based analysis and synthesis applied to the dynamics, guidance, and control of an autonomous undersea vehicle are presented. As the dynamic model for describing vehicle motion mathematically, the equations of motion are derived. The stability derivatives in the equations of motion are determined by a simulation-based technique using computational fluid dynamics analysis. The dynamic model is applied to the design of the low-level control systems, offering model-based synthetic approach in dynamics and control applications. As an intelligent navigational strategy for undersea vehicles, we present the optimal guidance in environmental disturbances. The optimal guidance aims at the minimum-time transit of a vehicle in an environmental flow disturbance. In this paper, a newly developed algorithm for obtaining the numerical solution of the optimal guidance law is presented. The algorithm is a globally working procedure deriving the optimal guidance in any deterministic environmental disturbance. As a fail-safe tactic in achieving the optimal navigation in environments of moderate uncertainty, we propose the quasi-optimal guidance. Performances of the optimal and the quasi-optimal guidances are demonstrated by the simulated navigations in a few environmental disturbances.
\end{abstract}

\section{Introduction}

In this article, we present model-based analysis and synthesis applied to the dynamics, guidance, and control of an autonomous undersea vehicle (AUV). The vehicle dynamics is one of the most important concerns in designing and developing an AUV, while the guidance and control are the key issues in achieving the desired vehicle performance. Our approach deals with these individual but closely interrelated issues in a consistent way based on the model-based simulations. 
In our research, as the dynamic model of an AUV, we employ a set of equations of motion describing the coupled six-degree-of-freedom (6-DOF) behaviour in threedimensional (3D) space. In the linearized form of the equations of motion, to complete the dynamic model of an AUV, we have to determine the so-called stability derivatives or hydrodynamic coefficients. There are many well-established approaches for determining the stability derivatives of aerial vehicles [1,2] or marine vehicles [3], which are based on either experiment or theoretical prediction. While the experimental approach allows direct measurement of the fluid dynamic forces and moments acting on the vehicle, it requires a large amount of time, labour, expense, as well as an experimental facility. On the other hand, a few state-of-the-art techniques are now available for predicting the stability derivatives theoretically [4-6]. Most of them, however, are specialized for deriving the stability derivatives for the dynamics of conventional airplanes [4,5] or ships [6], making them hard to directly apply to the modelling problems related to the dynamics of a specific AUV. In this respect, we introduce a general-purpose technique for deriving the dynamic model of an undersea vehicle, primarily depending on the computational fluid dynamics (CFD) analysis.

The derived dynamic model is directly applied to the model-based design of the motion control systems of an AUV. Two proportional-integral-derivative (PID) type low-level controllers are employed to make a vehicle follow the desired trajectories in the longitudinal and lateral planes, represented as time sequences of the depth (altitude) and heading.

As an intelligent high-level control of AUVs, a strategy of optimal guidance is presented. The optimal guidance proposed in this research is the minimum-time guidance in sea current environments, allowing a vehicle to reach a destination with the minimum travel time. When the power consumption of an AUV is controlled to be constant throughout the navigation, the navigation time is directly proportional to the total energy consumption. Released from the umbilical cable, an AUV has to rely on restricted energy stores during an undersea mission. Therefore, for an AUV, minimizing navigation time offers an enhanced potential for vehicle safety and mission success rate. We present a newly developed numerical procedure for deriving the optimal heading reference, by tracking which vehicle achieves the minimum-time navigation in a given sea current disturbance. The proposed procedure is systematic and seeks the solution in a global manner in any deterministic current field, whether stationary or time-varying. Moreover, unlike other path-finding algorithms, such as dynamic programming (DP) or generic algorithms (GAs) [7-9], our procedure does not require a computation time increase for the time-varying problems.

In real environments of AUV navigation, there are some factors that can cause failure in realizing the proposed optimal guidance strategy [10,11]. Some examples are environmental uncertainties, severe sensor noises, or temporally faulty actuators. Though these risk factors significantly affect the realization of optimality in actual sea navigation, they have not been seriously treated in most of related literatures. In this article, we present the concept of quasioptimality as a fail-safe strategy for realizing the proposed optimal navigation.

\section{An AUV "R-One"}

In this article, we practice our strategy in dynamics, guidance, and control on the AUV "ROne," a long-range cruising type AUV, developed by the Institute of Industrial Science (IIS), the University of Tokyo [12]. Figure 1 shows the overall layout of R-One. 


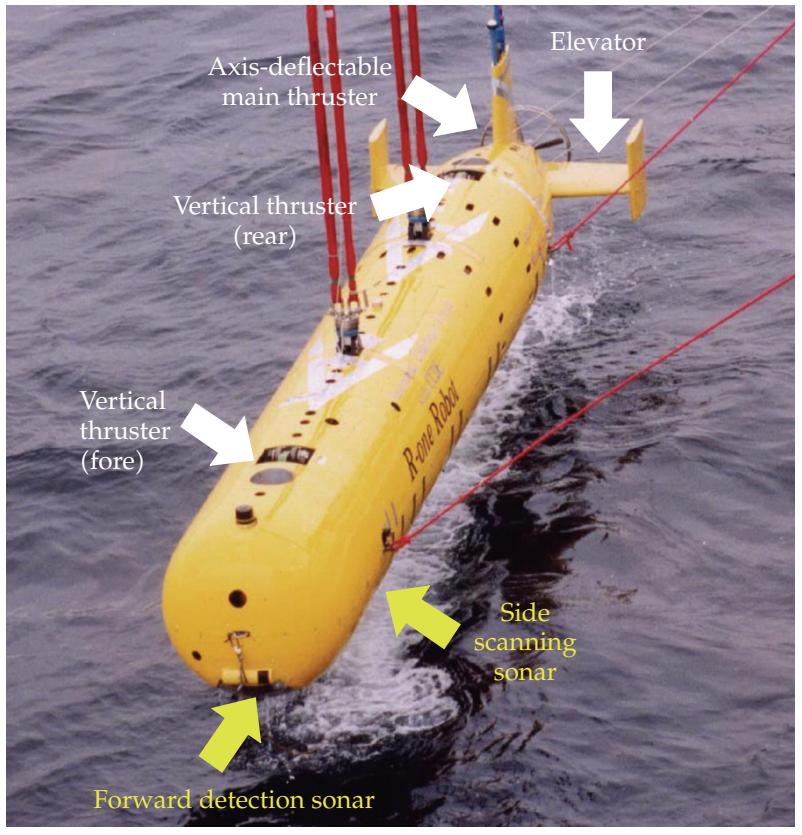

Figure 1: Overall layout of the long-range cruising type AUV R-One.

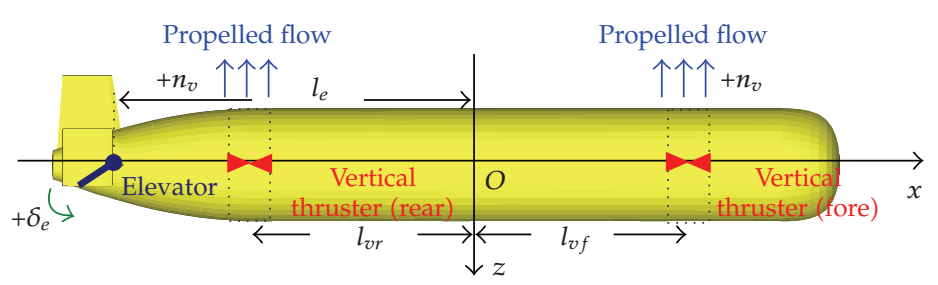

(a)

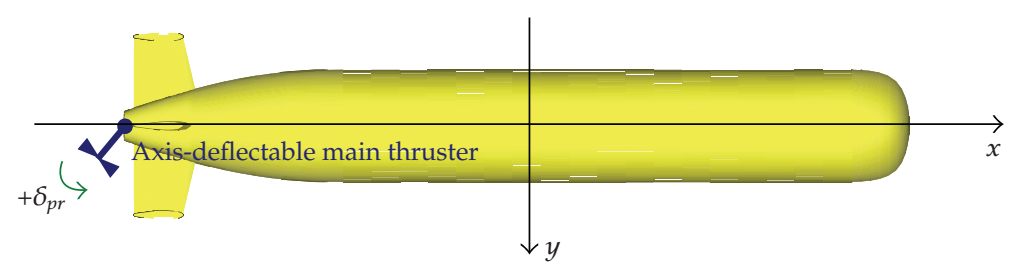

(b)

Figure 2: Coordinate system and actuator actions in describing the dynamics of R-One. The coordinate system takes its origin at the center of gravity of the vehicle. The $n_{v}$ is the rpm of fore and rear vertical thrusters. The $\delta_{e}$ is the elevator deflection. The $\delta_{p r}$ is the deflected amount of the main thruster axis.

Figure 2 shows the coordinate system and the actions of the actuators installed in the R-One. The axis-deflectable main thruster keeps or changes the vehicle's kinematic states in the horizontal plane. Two elevators and two vertical thrusters play the same role in the vertical plane. 


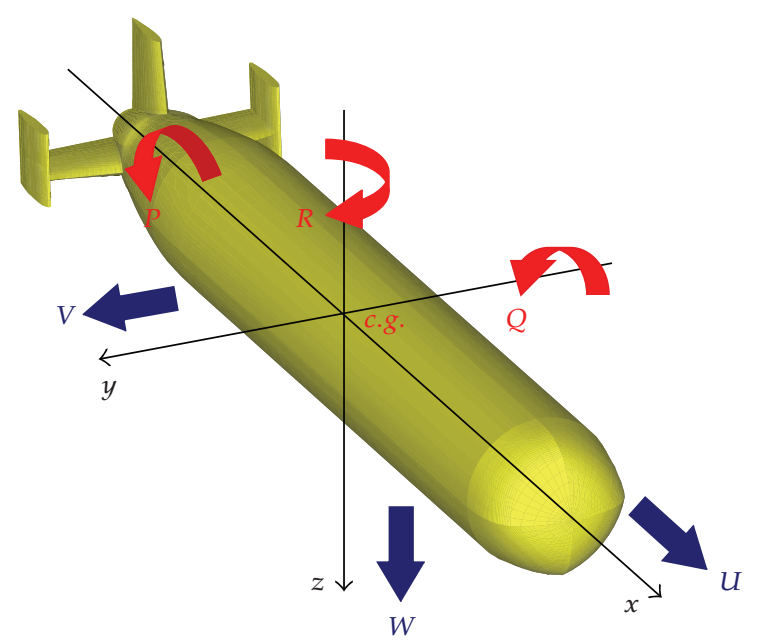

Figure 3: Body-fixed coordinate system with linear and angular velocity components.

\section{Modelling Vehicle Dynamics}

\subsection{Equations of Motion for Vehicle Dynamics.}

The equations of motion describing the vehicle motion mathematically can be derived from the conservation law of the linear and the angular momenta with respect to the inertial frame of $[1,2]$. The equations of motion (3.1) describing the 6-DOF motion of an AUV are defined with respect to the body-fixed frame of reference shown in Figure 3, in which the origin is taken at the vehicle's center of gravity. The procedures for deriving the equations of motion of an aerial vehicle which is quite similar to $(3.1)$ are found in $[1,2]$. It should be noted here however, that in deriving (3.1) by referring to the equations of motion for the aerial vehicles shown in $[1,2]$, the hydrostatic loads which do not appear in the flight dynamics have to be additionally involved

$$
\begin{gathered}
m(\dot{U}+Q W-R V)=-(m-\rho \nabla) g \sin \Theta+X, \\
m(\dot{V}+R U-P W)=(m-\rho \nabla) g \cos \Theta \sin \Phi+Y, \\
m(\dot{W}+P V-Q U)=(m-\rho \nabla) g \cos \Theta \cos \Phi+Z, \\
I_{x x} \dot{P}-I_{x z} \dot{R}-I_{x z} P Q+\left(I_{z z}-I_{y y}\right) Q R=\rho \nabla g z_{B} \cos \Theta \sin \Phi+L, \\
I_{y y} \dot{Q}+\left(I_{z z}-I_{z z}\right) R P+I_{x z}\left(P^{2}-R^{2}\right)=\rho \nabla g z_{B} \sin \Theta+M, \\
-I_{x z} \dot{P}+I_{z z} \dot{R}+\left(I_{y y}-I_{x x}\right) P Q+I_{x z} Q R=N .
\end{gathered}
$$

In (3.1), $U, V, W$, and $P, Q, R$ are the $x, y, z$ components of linear and angular velocities. $\nabla, m$, and $I$ represent volume, mass, and mass moments or products of inertia of a vehicle, and $\rho$ and $g$ are constants expressing water density and gravitational acceleration. Hydrodynamic forces and moments are represented by $X, Y, Z$, and $L, M, N$, each of which is the component in the direction of $x, y, z . \Phi, \Theta$ and $\Psi$ are so-called Euler angles to be 
defined in the coordinate transformation between the body-fixed and the inertial frames of reference. The $z_{B}$ is the $z$-directional displacement of the buoyancy center of the vehicle. The equations of motion are frequently linearized for use in stability and control analysis as mentioned in [1-3]. The following equations are the linearized forms of (3.1), in which $u, v, w, p, q, r, \phi, \theta$, and $\psi$ denote small amounts of velocities, and angular velocities and displacements, perturbed from their reference values, which are expressed by their uppercase letters

$$
\begin{gathered}
m\left(\dot{u}+q W_{0}\right)=-\theta(m-\rho \nabla) g \cos \theta_{0}+X, \\
m\left(\dot{v}+r U_{0}-p W_{0}\right)=\phi(m-\rho \nabla) g \cos \theta_{0}+Y, \\
m\left(\dot{w}-q U_{0}\right)=-\theta(m-\rho \nabla) g \sin \theta_{0}+Z, \\
I_{x x} \dot{p}-I_{x z} \dot{r}=\phi \rho \nabla g z_{B} \cos \theta_{0}+L, \\
I_{y y} \dot{q}=\theta \rho \nabla g z_{B} \cos \theta_{0}+M, \\
-I_{x z} \dot{p}+I_{z z} \dot{r}=N, \\
\dot{\phi}=p+r \tan \theta_{0}, \\
\dot{\theta}=q, \\
\dot{\psi}=r \sec \theta_{0} .
\end{gathered}
$$

In general, to complete the linearized equations of motion for use in stability and control analysis, hydrodynamic loads are expanded and linearized on the assumption that they are functions of the instantaneous values of the perturbed velocities, accelerations, and control inputs. Thus, the expanded expressions of the hydrodynamic loads are obtained in the form of a Taylor series in these variables, which is linearized by discarding all the higher-order terms. For example, $X$ is expanded as

$$
X=X_{u} u+X_{w} w+X_{\dot{u}} \dot{u}+X_{n_{m}} n_{m}
$$

where

$$
X_{u}=\left(\frac{\partial X}{\partial u}\right)_{0}, \quad X_{w}=\left(\frac{\partial X}{\partial w}\right)_{0}, \ldots
$$

The subscript zero in (3.3a) indicates a reference condition where the derivatives are evaluated. In (3.3a) and (3.3b), derivatives such as $X_{u}$ or $X_{w}$ are called stability derivatives [1-5]. By expanding all the external hydrodynamic loads introducing stability derivatives 
of their dynamic correlations, the equations of motion (3.2) are expressed by means of the stability derivatives as

$$
\begin{gathered}
\left(m-X_{\dot{u}}\right) \dot{u}+m W_{0} \dot{\theta}-X_{u} u-X_{w} w+\theta(\rho \nabla-m) g \cos \theta_{0}=X_{n_{m}} n_{m} \\
\left(m-Z_{\dot{w}}\right) \dot{w}-Z_{\dot{q}} \dot{q}-Z_{u} u-Z_{w} w-\left(m U_{0}+Z_{q}\right) q-\theta(\rho \nabla-m) g \sin \theta_{0}=Z_{n_{v}} n_{v}+Z_{\delta e} \delta_{e} \\
-M_{\dot{w}} \dot{w}+\left(I_{y y}-M_{\dot{q}}\right) \dot{q}-M_{u} u-M_{w} w-M_{q} q-\theta \rho \nabla z_{B} \cos \theta_{0}=-Z_{n_{v}} l_{v f} n_{v}+Z_{n_{v}} l_{v r} n_{v}+Z_{\delta e} l_{e} \delta_{e}, \\
\left(m-Y_{\dot{v}}\right) \dot{v}-Y_{\dot{r}} \dot{r}-Y_{v} v-\left(m W_{0}+Y_{p}\right) p+\left(m U_{0}-Y_{r}\right) r=Y_{\delta_{p r}} \delta_{p r} \\
-L_{\dot{v}} \dot{v}+\left(I_{x x}-L_{\dot{p}}\right) \dot{p}-\left(I_{x z}+L_{\dot{r}}\right) \dot{r}-L_{v} v-L_{p} p-L_{r} r-\phi \rho \nabla g z_{B} \cos \theta_{0}=L_{\delta_{p r}} \delta_{p r} \\
-N_{\dot{v}} \dot{v}+\left(I_{x z}+N_{\dot{p}}\right) \dot{p}+\left(I_{z z}-N_{\dot{r}}\right) \dot{r}-N_{v} v-N_{p} p-N_{r} r=N_{\delta_{p r}} \delta_{p r}, \\
\dot{\phi}=p+r \tan \theta_{0}, \\
\dot{\theta}=q \\
\dot{\psi}=r \sec \theta_{0}
\end{gathered}
$$

where $n_{m}$ represents the rpm of the main thruster.

\subsection{Evaluation of Stability Derivatives by CFD Analyses.}

As noticeable in (3.3), within the framework of small perturbation theory, constructing the dynamic model is, in effect, reduced to the determination of the stability derivatives defined in the linearized equations of motion. The most commonly and widely employed approaches for evaluating the stability derivatives are the wind tunnel test for aerial vehicles and the towing tank test for marine vehicles [1-6]. These experimental approaches, however, require a huge experimental facility and a large workforce, which makes them expensive and laborious, even when the test is for a single model. In this article, we present a modelbased approach for evaluating the stability derivatives. In the approach, dominant stability derivatives are evaluated from the hydrodynamic loads which are obtained by CFD analyses. When we are to evaluate the value of $X_{u}$ in (3.3) defined at a reference speed of $U_{0}$, for example, we conduct CFD analyses repeatedly at the cruising speeds of $U_{0}\left(\begin{array}{l}1 \pm \eta \\ \text {, }\end{array}\right.$ where $U_{0}$ is the reference cruising speed and $\eta$ is the perturbation ratio of $U_{0}$. By taking central difference approximation of $X$ with respect to $u$ by using the $X$ values obtained at $U_{0}(1 \pm \eta)$, we can derive $X_{u}$ defined at $U_{0}$. However, while the majority of dominant stability derivatives are able to be evaluated by this technique, there are other stability derivatives which are not. For such stability derivatives, the simplified estimation formulae proposed in the field of flight dynamics [1,2] are modified and applied.

In our CFD analyses, we used a commercial fluid dynamics solver called "Star-CD," developed by CD-adapco [13]. The Star-CD is a Navier-Stokes solver based on the finite difference numerical scheme. Like other famous commercial CFD solvers such as FLUENT or ANSYS, Star-CD also has shown numerous field application results that it replicates experimental model results with acceptably fine accuracy [14, 15]. The Star-CD derives 

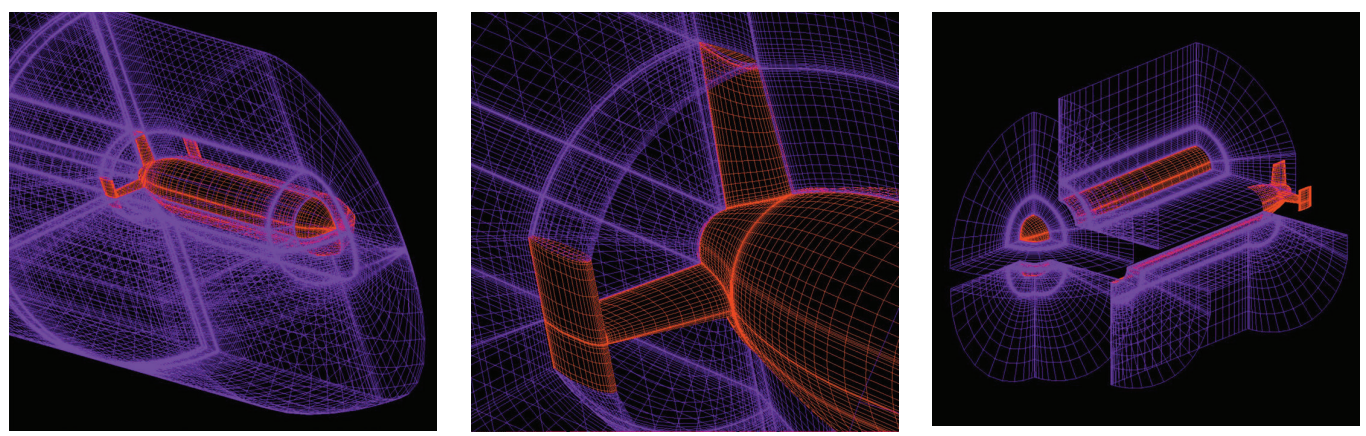

Figure 4: Grid system for CFD analyses of flow field around the R-One. The entire grid system is completed by assembling individually generated subgrid blocks.

the numerical solution by pressure-implicit split-operator (PISO) algorithm, which is a wellknown, robust scheme with predictor-corrector steps. In our CFD analyses, the problemspecific high Reynolds number $(\mathrm{Re})$ requires a proper turbulence model. In using Star-CD, we selected the Reynolds-Averaged Navier-Stokes (RANS) turbulence model, which is one of the most widely used turbulence model in engineering applications of moderate turbulent conditions [16].

Figure 4 shows the grid system for evaluating the hydrodynamic loads by CFD analyses. To generate a computationally robust, structured grid system adapting to the complicated aftbody geometry of the vehicle, we employed a grid generation technique called the multiblock method [17].

Not only estimating the drag force of acceptable accuracy is the primary concern in our CFD analyses for deriving the stability derivatives, it also serves as the most fundamental measure to evaluate a CFD solver [14-16]. After completing the hull structure, drag forces acting on the R-One at three cruising speeds were investigated by means of the towing tank tests [12]. In Figure 5, two drag curves, obtained by CFD calculations and tank tests, are shown together. The drag curves shown are quadratic interpolations of the raw data set of drags, calculated and measured at the cruising speeds of $1.03,1.54$, and $2.06 \mathrm{~m} / \mathrm{s}$, respectively. Validity of the quadratic interpolation is based on the fact that within the small Re interval, drag of an immersed body has quadratic dependency on its advance speed [18]. In Figure 5, the gradients of two drag curves are also expressed. As seen in the figure, drags obtained by CFD calculations are more or less excessive than the ones by tank tests. However, it is noted that the gradients of drags show close similarity between CFD analyses and tank tests, which advocates our approach to evaluating the stability derivatives principally by means of the CFD analyses.

Figure 6 shows the pressure distribution with a few selected streamlines along the body surface of R-One. By integrating the pressure over the entire body surface, hydrodynamic loads are obtained.

It is generally known and also noticeable from (3.3) that, according to the coupling relation, linearized equations of motion are split into two independent groups: longitudinal equations for surge, heave, and pitch, and lateral equations for sway, roll, and yaw [1-3]. In Tables 1(a) and 1(b), the longitudinal and lateral stability derivatives appearing in (3.3) are summarized. By substituting all stability derivatives in (3.3) with their corresponding numerical values in the tables, the dynamic model of R-One is completed. 


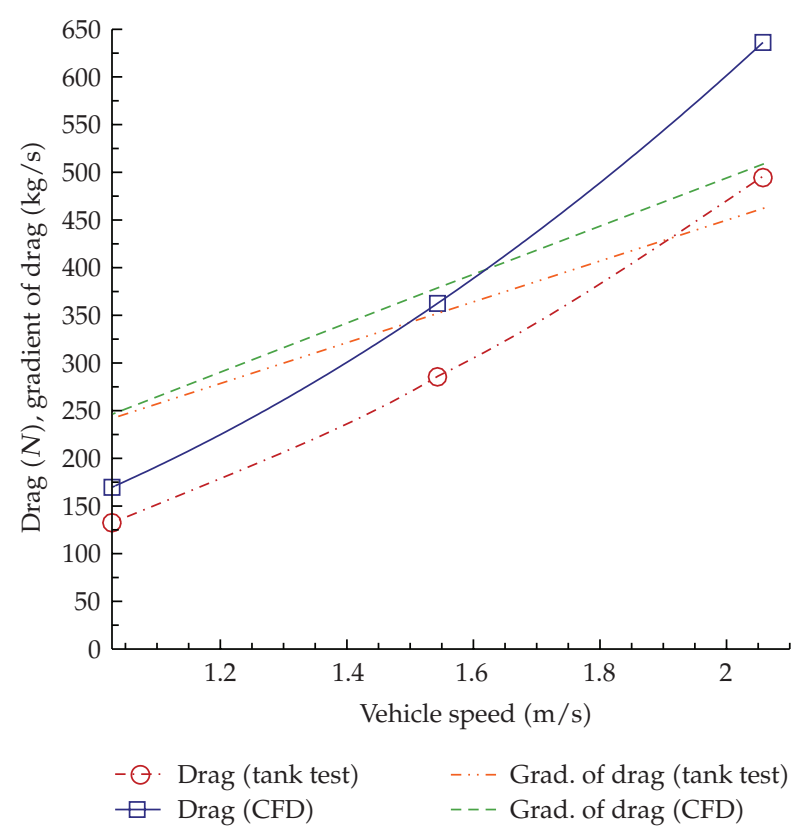

Figure 5: Curves of drags and gradient of drags.

Table 1: (a) Stability derivatives in the longitudinal equations of motion for R-One. (b) Stability derivatives in the lateral equations of motion for R-One.

(a)

\begin{tabular}{|c|c|c|c|c|c|}
\hline$\overline{X_{u}}$ & $(\mathrm{~kg})$ & -237.65 & $Z_{u}$ & $(\mathrm{~kg} / \mathrm{s})$ & -64.41 \\
\hline$Z_{\dot{w}}$ & (kg) & -2152.10 & $Z_{w}$ & $(\mathrm{~kg} / \mathrm{s})$ & -2819.64 \\
\hline$Z_{\dot{q}}$ & $(\mathrm{~kg} \cdot \mathrm{m})$ & -25547.00 & $Z_{q}$ & $(\mathrm{~kg} \cdot \mathrm{m} / \mathrm{s})$ & -11360.06 \\
\hline$M_{\dot{u}}$ & $(\mathrm{~kg} \cdot \mathrm{m})$ & 0.00 & $M_{u}$ & $(\mathrm{~kg} \cdot \mathrm{m} / \mathrm{s})$ & 0.00 \\
\hline$M_{\dot{w}}$ & $(\mathrm{~kg} \cdot \mathrm{m})$ & -1928.80 & $M_{w}$ & $(\mathrm{~kg} \cdot \mathrm{m} / \mathrm{s})$ & 870.36 \\
\hline$M_{\dot{q}}$ & $\left(\mathrm{~kg} \cdot \mathrm{m}^{2}\right)$ & -153400.00 & $M_{q}$ & $\left(\mathrm{~kg} \cdot \mathrm{m}^{2} / \mathrm{s}\right)$ & -39351.25 \\
\hline$X_{u}$ & $(\mathrm{~kg} / \mathrm{s})$ & -364.37 & $Z_{\delta_{e}}$ & $\left(\mathrm{~kg} \cdot \mathrm{m} / \mathrm{s}^{2}\right)$ & -3168.10 \\
\hline$X_{w}$ & $(\mathrm{~kg} / \mathrm{s})$ & 64.72 & $M_{\delta_{e}}$ & $\left(\mathrm{~kg} \cdot \mathrm{m}^{2} / \mathrm{s}^{2}\right)$ & -10974.31 \\
\hline$X_{q}$ & $(\mathrm{~kg} \cdot \mathrm{m} / \mathrm{s})$ & 0.00 & & & \\
\hline \multicolumn{6}{|c|}{ (b) } \\
\hline$Y_{\dot{v}}$ & $(\mathrm{~kg})$ & -4653.77 & $Y_{r}$ & $(\mathrm{~kg} \cdot \mathrm{m} / \mathrm{s})$ & 3931.06 \\
\hline$Y_{\dot{r}}$ & $(\mathrm{~kg})$ & 746.74 & $L_{v}$ & $(\mathrm{~kg} \cdot \mathrm{m} / \mathrm{s})$ & -515.99 \\
\hline$L_{\dot{v}}$ & $(\mathrm{~kg} \cdot \mathrm{m})$ & -1.31 & $L_{p}$ & $\left(\mathrm{~kg} \cdot \mathrm{m}^{2} / \mathrm{s}\right)$ & -1165.25 \\
\hline$L_{\dot{p}}$ & $\left(\mathrm{~kg} \cdot \mathrm{m}^{2}\right)$ & 3.81 & $L_{r}$ & $\left(\mathrm{~kg} \cdot \mathrm{m}^{2} / \mathrm{s}\right)$ & 1500.45 \\
\hline$L_{\dot{r}}$ & $\left(\mathrm{~kg} \cdot \mathrm{m}^{2}\right)$ & -3.35 & $N_{v}$ & $(\mathrm{~kg} \cdot \mathrm{m} / \mathrm{s})$ & -4054.37 \\
\hline$N_{\dot{v}}$ & $(\mathrm{~kg} \cdot \mathrm{m})$ & -554.21 & $N_{p}$ & $\left(\mathrm{~kg} \cdot \mathrm{m}^{2} / \mathrm{s}\right)$ & -1.02 \\
\hline$N_{\dot{p}}$ & $\left(\mathrm{~kg} \cdot \mathrm{m}^{2}\right)$ & -1.19 & $N_{r}^{r}$ & $\left(\mathrm{~kg} \cdot \mathrm{m}^{2} / \mathrm{s}\right)$ & -13704.63 \\
\hline$N_{\dot{r}}$ & $\left(\mathrm{~kg} \cdot \mathrm{m}^{2}\right)$ & -15934.27 & $Y_{\delta_{\mathrm{pr}}}$ & $\left(\mathrm{kg} \cdot \mathrm{m} / \mathrm{s}^{2}\right)$ & -399.04 \\
\hline$Y_{v}$ & $(\mathrm{~kg} / \mathrm{s})$ & -1809.97 & $L_{\delta_{\mathrm{pr}}}$ & $\left(\mathrm{kg} \cdot \mathrm{m}^{2} / \mathrm{s}^{2}\right)$ & 0.00 \\
\hline$Y_{p}$ & $(\mathrm{~kg} \cdot \mathrm{m} / \mathrm{s})$ & 0.00 & $N_{\delta_{\mathrm{pr}}}$ & $\left(\mathrm{kg} \cdot \mathrm{m}^{2} / \mathrm{s}^{2}\right)$ & 1677.38 \\
\hline
\end{tabular}



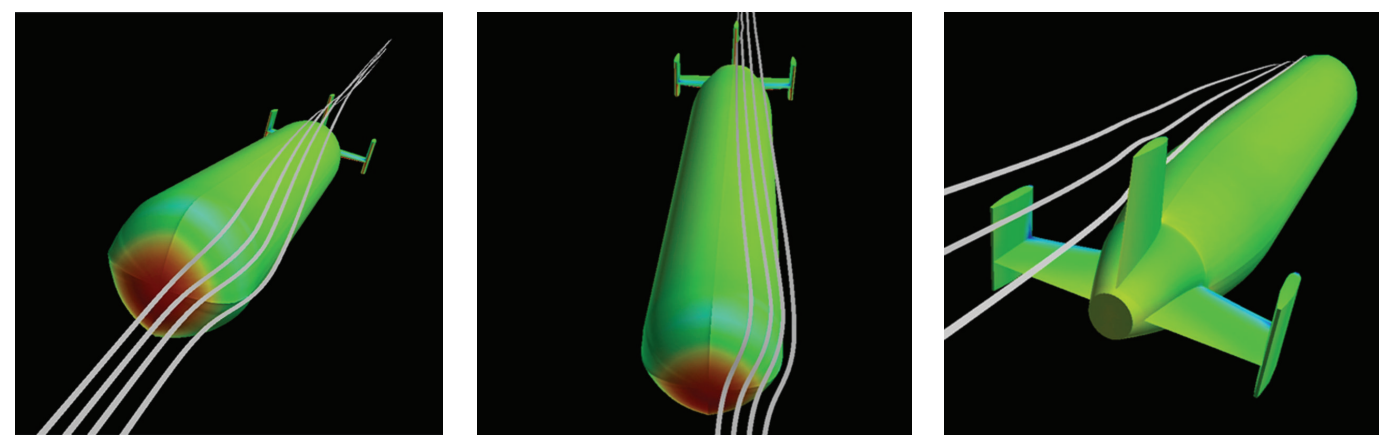

Figure 6: Visualized results of a CFD analysis.

\subsection{Vehicle Motion Simulation}

State-space forms of the longitudinal and the lateral equations of motion for R-One, completed by assigning the numerical values in Table 1 to corresponding stability derivatives in (3.3), are represented as follows.

(i) Longitudinal equations of motion for R-One:

$$
\left[\begin{array}{c}
\dot{u} \\
\dot{w} \\
\dot{q} \\
\dot{\theta}
\end{array}\right]=\left[\begin{array}{cccc}
-0.0786 & 0.0140 & 0 & 0.0145 \\
-0.0103 & -0.4725 & 0.2465 & 0.0610 \\
0.0001 & 0.0108 & -0.2420 & -0.0156 \\
0 & 0 & 1 & 0
\end{array}\right]\left[\begin{array}{c}
u \\
w \\
q \\
\theta
\end{array}\right]+\left[\begin{array}{ccc}
0.0554 & 0 & 0 \\
0 & 0.0027 & -0.2169 \\
0 & -0.0001 & -0.0684 \\
0 & 0 & 0
\end{array}\right]\left[\begin{array}{l}
n_{m} \\
n_{v} \\
\delta_{e}
\end{array}\right] .
$$

(ii) Lateral equations of motion for R-One:

$$
\left[\begin{array}{c}
\dot{v} \\
\dot{p} \\
\dot{r} \\
\dot{\phi}
\end{array}\right]=\left[\begin{array}{cccc}
-0.2097 & 0.0053 & -0.5388 & 0.0112 \\
-4.7444 & -11.2192 & 16.1215 & -23.6516 \\
-0.1185 & 0.0643 & -1.1931 & 0.1357 \\
0 & 1 & 0 & 0
\end{array}\right]\left[\begin{array}{c}
v \\
p \\
r \\
\phi
\end{array}\right]+\left[\begin{array}{c}
-0.0388 \\
-0.0948 \\
0.0634 \\
0
\end{array}\right] \delta_{p r} .
$$

By solving (3.5a) and (3.5b) in the time domain with appropriate initial conditions and actuator inputs, motion responses of the R-One are computed. In the inertial navigation system (INS) installed in R-One, not only vehicle kinematics but also time sequences of the actuator inputs during an undersea mission are recorded. In Figure 7, simulated vehicle trajectories are compared with actual vehicle trajectories recorded during the Teisi knoll survey mission [19]. As noticeable from the figure, the dynamic model of R-One implemented by our model-based approach provides motion responses exhibiting sufficiently good agreement between the simulated and actual vehicle trajectories. 


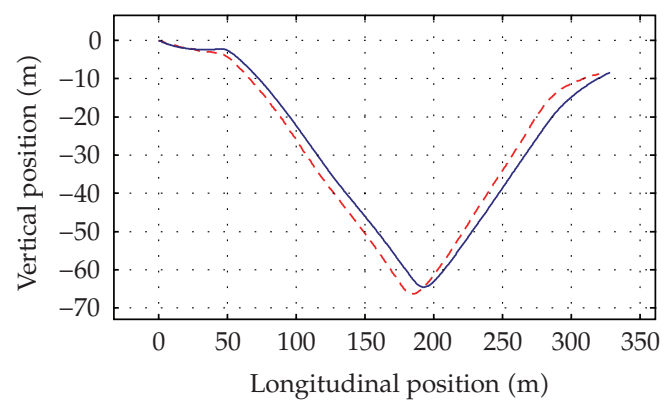

(a)

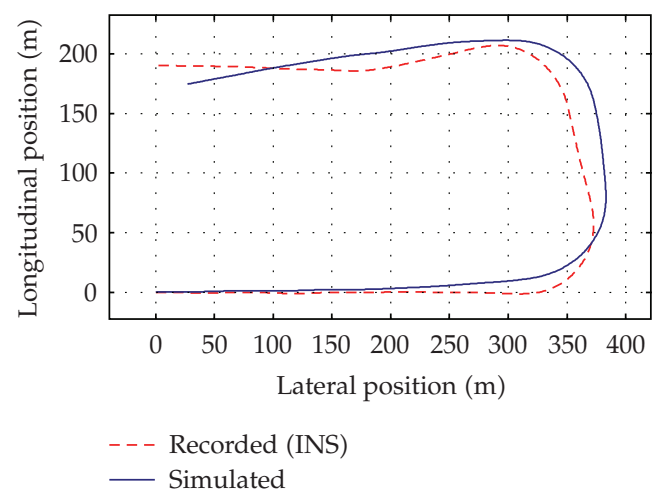

(c)

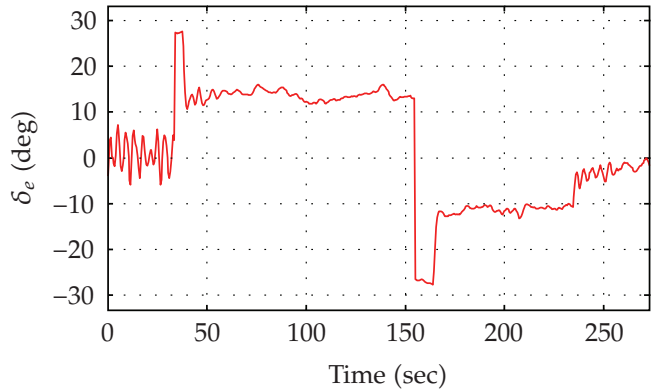

(b)

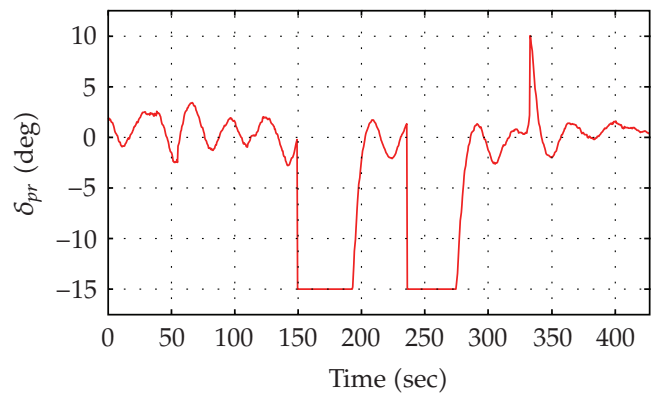

(d)

Figure 7: Simulated and actual vehicle trajectories (a and c) generated from the corresponding actuator inputs (b and $d)$.

\section{Tracking Control Design}

The controller implemented for the motion control of R-One is based on the PID compensation. Needless to say that, PID-type controller is the most commonly and widely used controller for most artificial control systems. However, in designing a PID controller, precise plant dynamics is a key prerequisite to ensuring acceptably good control performance. Deriving a precise plant dynamics is not easy in some cases. For this reason, during the past three decades, a few significant attempts have been made to provide controller models that do not depend on a precise description of the plant model in its design [20-22]. Neural network (NN) controllers based on the self-organizing map or fuzzy logic controllers are the most famous ones in such attempts [21,22]. In order to derive a practically useful controller by NN or fuzzy logic, however, we have to ensure huge random diversity in training data. This is a very difficult task in a real world problem, because, in general, we do not have any definitive guidelines for deciding whether the prepared training data is biased or not $[22,23]$.

To change or keep the kinematic states of the vehicle, two independent low-level controls were implemented in the R-One: the depth (altitude) control for the longitudinal motion and the heading control for the lateral motion. Configurations of the depth and the heading controls are depicted in Figures 8(a) and 8(b).

To build the mathematical models for the control systems shown in the Figure 8, transfer functions of $p(s) / \delta_{e}(s), w(s) / \delta_{e}(s)$, and $r(s) / \delta_{p r}(s)$ are extracted from (3.5a) and 


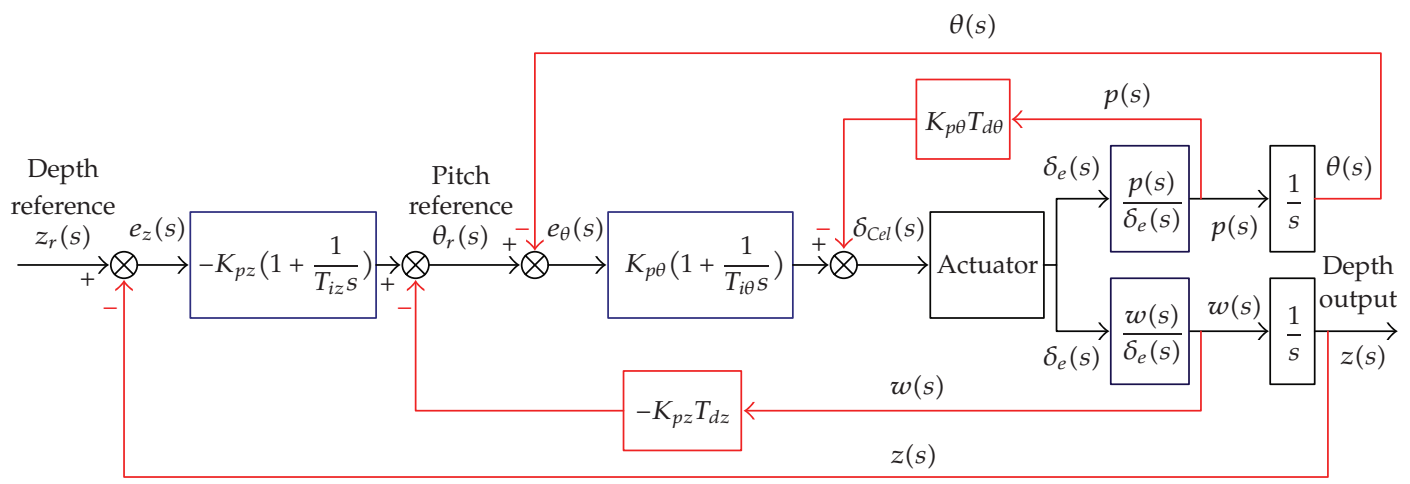

(a)

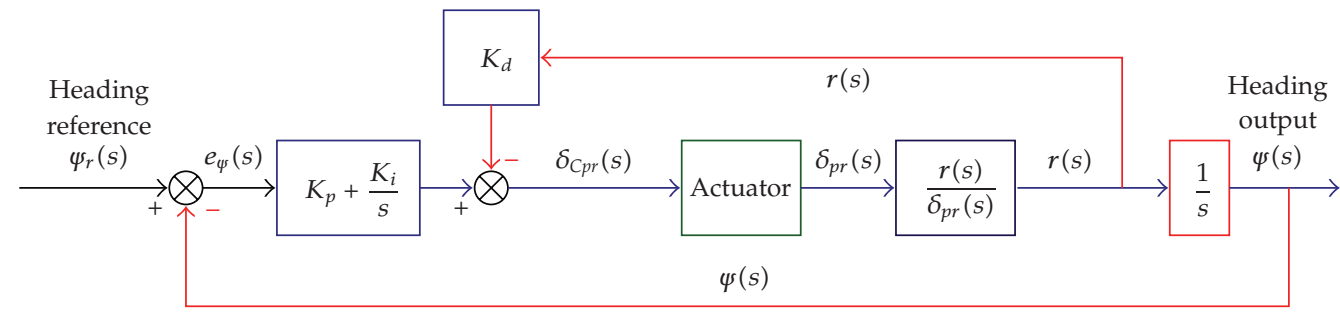

(b)

Figure 8: (a) Configuration of the depth (altitude) control system for R-One. (b) Configuration of the heading control system for R-One.

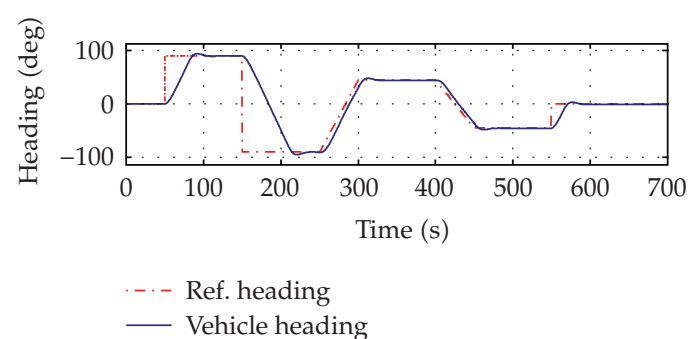

(a)

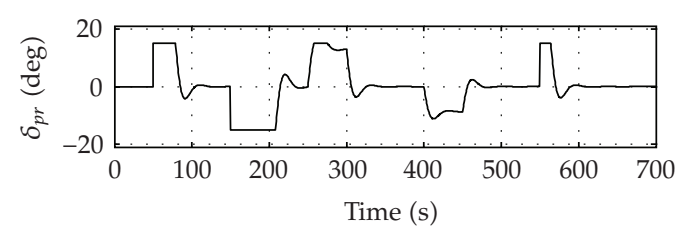

(b)

Figure 9: Simulated heading tracking control (a) with corresponding actuator input (b).

(3.5b). Then, PID-tuning is carried out to determine the optimal values of controller gains from the standpoint of system robustness and swiftness of response. In determining the optimal gain values, we used the model-based control system design tool called "SISO Design Tool," offered by the "Control System Toolbox" included in "Matlab" [24].

An example of the performance result for the designed control systems is shown in Figure 9, where it is clearly seen that the designed heading controller lets the vehicle follow the heading reference with sufficient swiftness and small overshoot. 


\section{Optimal Guidance of AUV}

\subsection{Background}

The sea environment contains several disturbances, such as surface waves, wind, and sea currents. Among them, the sea current is known to be the most significant disturbance for the dynamics of an undersea vehicle as it directly interacts with the vehicle motion $[3,8,11]$. Considering the guidance problem to make a vehicle transit to a given destination in a region of sea current, it is quite natural that there arises a navigation time difference according to the selection of an individual navigation trajectory. The problem of the minimum-time vessel guidance in a region of current flow has interested people as long years ago as ancient Greece [25]. However, since the problem requires a minimization technique of the functionals, it had hardly been treated mathematically until the advent of the calculus of variations. On the basis of this mathematical tool, Bryson and Ho [26] derived the minimum-time guidance law of a surface vessel in a region of a surface current flow. Though the law is an optimal controller of explicit form, obtaining its solution is not easy since it actually is a so-called two-point boundary value problem. As an ad hoc approach for the minimum-time navigation problem in a linearly varying, shear flow-like current distribution presented by Lewis and Syrmos, a graphical solution finding technique has been presented [25]. As is naturally expected, however, such an approach is problem-specific and lacks universality in its applicability. Papadakis and Perakis [8] treated the minimum-time routing problem of a vessel moving in a wave environment. In their approach, by subdividing the navigation region into several subregions of different sea states, the path for the optimal routing is obtained by the DP approach. Aside from the difficulties in constructing a numerical solution procedure for their approach, it has a problem that the solution significantly depends on the features of regional subdivision. As a completely discrete and nonlinear approach, the cell mapping technique was applied to derive the minimum-time tracking trajectory to capture a moving target in a deterministic vortex field [27]. It, however, has the same problem of regional subdivision as is inherent in the approach by Papadakis and Perakis [8], which might lead to the divergence due to numerical instability.

In this research, we propose a newly developed procedure for obtaining the numerical solution of the optimal guidance law, which achieves the minimum-time navigation of a vehicle in a given current field. The algorithm of our solution procedure is simple but consistently applicable to any current field if only the distribution of which is deterministic. As a fault-tolerable strategy for putting the proposed optimal navigation into practice, the concept of quasioptimality is introduced. The basic idea of the quasioptimal navigation is quite simple and, in effect, consists of the on-site feedbacks of the optimal guidance revisions.

\subsection{The Optimal Guidance Law}

In our optimal guidance problem, we employed the guidance law presented by Bryson and Ho [26] as

$$
\dot{\psi}=\sin ^{2} \psi \frac{\partial v_{c}}{\partial x}+\frac{1}{2}\left(\frac{\partial u_{c}}{\partial x}-\frac{\partial v_{c}}{\partial y}\right) \sin 2 \psi-\cos ^{2} \psi \frac{\partial u_{c}}{\partial y}
$$

where $\psi$ represents the vehicle heading as defined in Figure 10 , and $u_{c}, v_{c}$ are $x, y$ components of the sea current velocity. The detailed procedure of deriving (5.1) is well explained in [11]. 


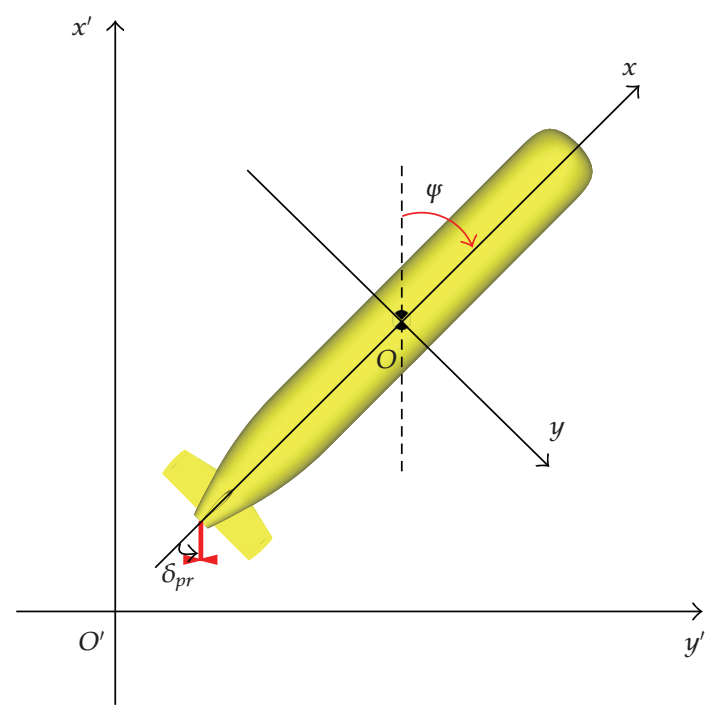

Figure 10: Coordinate system and definition of the heading for describing the optimal guidance problem. $O^{\prime}-x^{\prime} y^{\prime}$ is the inertial frame of reference, while $O-x y$ is the body-fixed coordinate system.

Though Bryson and Ho derived (5.1) on the assumption of a stationary flow condition, we have shown that it is also valid for time-varying currents, like tidal flows [11]. Equation (5.1) is a nonlinear ordinary differential equation of an unspecified vehicle heading $\psi(t)$. Though it seems that the solution would be readily obtainable by using a suitable numerical scheme, such as Runge-Kutta, there still remains a significant shortfall: while (5.1) defines an initial value problem, its solution cannot be obtained with an arbitrarily assigned initial heading. If we solve (5.1) with an arbitrary initial value of $\psi$, a vehicle following the solution of (5.1) as the heading reference does not arrive at the destination. This is because (5.1) is, in fact, a two-point boundary value problem, the correct initial value of which constitutes a part of the solution.

\subsection{Numerical Solution Procedure}

To obtain the solution of the two-point boundary value problems, an iterative solution procedure is generally used, such as "shooting" or "relaxation" [28]. Starting from an initial guess, solutions generated by these schemes are repeatedly adjusted to eliminate the discrepancies between the estimated and the desired boundary conditions at both endpoints. These schemes, however, strongly rely on the initial guess, inappropriate assignment of which may lead to a local solution or divergence [28]. In this article, we present a numerical procedure to obtain the solution of (5.1), called "AREN," which stands for Arbitrary REference Navigation". In applying AREN, first we need to make a simulated navigation along any feasible trajectory, generated by an arbitrary guidance and terminating at the destination. We call this navigation the "reference navigation," because it is used as the reference in deriving the optimal navigation. In a reference navigation, the time it takes for the vehicle to reach the destination should be recorded, which we call the "reference navigation time," denoted by $t_{f \text { ref. }}$. As already mentioned, the only requirement for the reference navigation is letting the vehicle arrive at the destination. Therefore, a navigation following any trajectory shown in Figure 11 can be qualified as the reference navigation. 


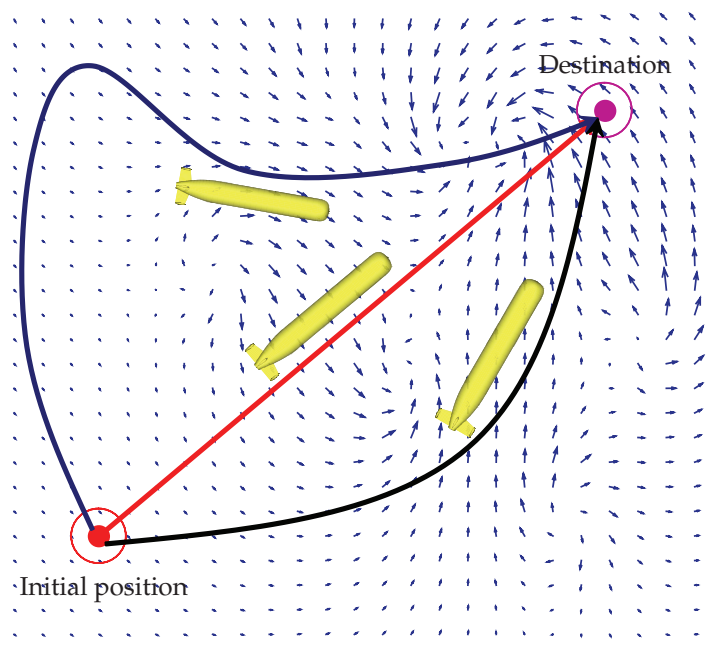

Figure 11: A few possible trajectories for the reference navigation.

In AREN, to search for the correct initial heading numerically, the interval of $0 \sim 2 \pi$ is divided by equally spaced $N-1$ subintervals as

$$
\psi_{0}^{(i)}=i \Delta \psi \quad \text { for } i=0,1, \ldots, N-1,
$$

where $\Delta \psi=2 \pi / N$. In (5.2), $\psi_{0}^{(i)}$ is the (i)th initial heading trial and $\Delta \psi$ is its increment, that is, the interval of initial heading trials. Next, for an initial heading trial $\psi_{0}^{(i)}$, we solve (5.1) in the time domain using an appropriate time marching scheme, which produces a simulated navigation starting from $\psi_{0}^{(i)}$. The navigation produced here is called the (i)th "trial navigation" adjoining $\psi_{0}^{(i)}$. Once the trajectory produced by a trial navigation passes through vicinity of the destination, it can be considered as a potential optimal navigation since the optimal guidance law (5.1) with the correct initial heading lets a vehicle reach the destination. Therefore, $N$ trial navigations starting from $N$ initial headings given in (5.2) are all possible candidates for the optimal navigation. In practice, however, discretization error in initial heading trials causes convergence error at the destination, so that the optimal navigation should be identified in an approximate manner. We define the "minimum distance," as the shortest distance between the destination and the trajectory generated by trial navigation. In Figure $12, l_{\mathrm{min}}^{(k-1)}, l_{\mathrm{min}}^{(k)}$, and $l_{\min }^{(k+1)}$ represent the minimum distances between the destination and the trajectories generated by $(k-1)$ th, $(k)$ th, and $(k+1)$ th trial navigations, respectively. If the minimum distance of the $(k)$ th trial navigation is smaller than any other minimum distance and thus satisfies (5.3), we choose it as the optimal navigation because in the $(k)$ th trial navigation, the vehicle approaches the destination with the smallest deviation among all trial navigations, that is, the optimal navigation candidates.

$$
l_{\min }^{(k)} \leq l_{\min }^{(i)}, \quad \text { for } i=0,1, \ldots, N-1 .
$$

However, in choosing the optimal navigation among the trial ones, there still remains a serious drawback: we have no idea how long we have to continue a trial navigation so as to 


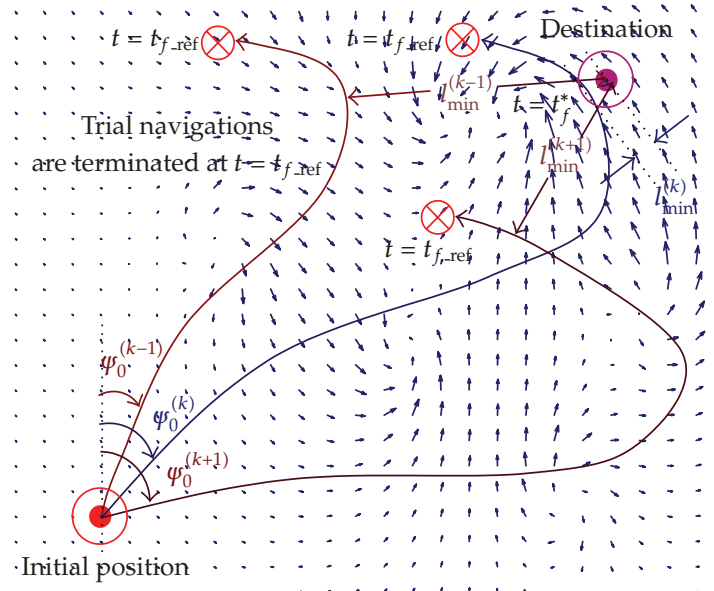

Figure 12: Trial navigations corresponding to a few discrete initial heading trials. The approximate optimal navigation converging to the destination is determined on the basis of the minimum distances. Note that all trial navigations are continued until $t=t_{f \text {-ref. }}$

determine its true minimum distance. This is where the reference navigation time prepared beforehand comes into play. It is apparent that the reference navigation is nonoptimal, since it is based on an arbitrary guidance law, only achieving the vehicle's arrival at the destination. Therefore, the reference navigation time must be larger or equal to that of the optimal navigation as

$$
O<t_{f}^{*} \leq t_{f \_ \text {ref }}
$$

where $t_{f}^{*}$ represents navigation time with the optimal guidance. It should be noted here that, according to the minimum principle, once we have started a trial navigation with an initial heading close to the optimal one, a vehicle should pass by the vicinity of the destination at a time surely smaller than $t_{f \text { _ref. }}$. In other words, the reference navigation time qualifies to be the upper limit of the simulation times of the trial navigations, in order to identify an optimal navigation among the trial ones. In Figure 12, among all trial navigations continued until $t=t_{f \text { ref }},(k)$ th trial navigation marks the smallest minimum distance, satisfying (5.3). Therefore, the $(k)$ th trial navigation is determined as the optimal navigation.

\section{Optimal Navigation Examples}

\subsection{The Reference Navigation}

As mentioned in the previous section, to implement the optimal guidance for an AUV navigation by AREN, it is necessary to make a reference navigation beforehand. The simplest guidance satisfying the vehicle's arrival at the destination may be proportional navigation (PN) $[25,26]$. In PN, vehicle heading is continuously adjusted so that the line of sight (LOS) is directed toward the target point. In our work, we employ PN as the reference navigation. 


\subsection{Optimal Navigation in a Shearing Flow}

The first numerical example in this research is an optimal navigation in a current disturbance of the linear shear flow, taken from Bryson and Ho [26]. The current velocity in this problem is described by

$$
\begin{gathered}
u_{c}(x, y)=0, \\
v_{c}(x, y)=-\frac{U_{c} x}{h},
\end{gathered}
$$

where $U_{c}$ and $h$ are set to be $1.544 \mathrm{~m} / \mathrm{s}$ and $100 \mathrm{~m}$, respectively. Starting from the initial position at $\left(x_{0}, y_{0}\right)=(-186 \mathrm{~m}, 366 \mathrm{~m})$, the vehicle is directed to move toward the destination at the origin in this example. Due to its simplicity, the current distribution of (6.1) allows derivation of the analytic optimal guidance law expressed as

$$
\begin{gathered}
\frac{x}{h}=\csc \psi-\csc \psi_{f}, \\
\frac{y}{h}=\frac{1}{2}\left[\csc \psi_{f}\left(\cot \psi-\cot \psi_{f}\right)+\cot \psi\left(\csc \psi_{f}-\csc \psi\right)+\log \frac{\csc \psi_{f}-\cot \psi_{f}}{\csc \psi-\cot \psi}\right],
\end{gathered}
$$

where $\psi_{f}$ is the vehicle heading at the final state.

Navigation trajectories are shown in Figure 13. In the reference navigation by PN, significant adverse drift happens at the initial stage, since within the region of $|x|>100 \mathrm{~m}$ current flow speed exceeds the vehicle speed relative to the water. The optimal guidance detours the vehicle across the upper half plane, taking advantage of the favorable current flow. Navigation times by PN and optimal guidance are 353.7 and $739.2 \mathrm{~s}$, respectively, indicating a $52 \%$ decrease in navigation time by the optimal guidance proposed.

\subsection{Optimal Navigation in a Time-Varying Flow}

The next numerical example is an optimal navigation in a time-varying current flow. In actual sea environments, for a lot of currents the direction and the magnitude of their velocities change continuously like tidal flows. As mentioned previously, we have proved that the optimal guidance law (5.1) is also valid for time-varying currents as well as for stationary ones. Therefore, once the flow velocity distribution in a navigation region is described as a function of the position and time, our numerical scheme is expected to be effective and thus realize the minimum-time navigation in a time-varying flow.

Navigation trajectories in an artificially made time-varying current are depicted in Figure 14. As shown in the figure, near the middle of the navigation region, the vehicle following the guidance of PN temporarily fails to proceed toward the destination due to severe drift caused by strong local current flow of adverse direction. The occurrence of such a disadvantage is prevented in the optimal navigation. By following the optimal guidance, the vehicle proceeds taking advantage of the favorable flows and avoids passing through the region in which the current flow is gradually changing to the strongly adverse one. In Figure $14(\mathrm{~b})$, it should be noted that at $623.0 \mathrm{~s}$, having been released from the severe drift, 


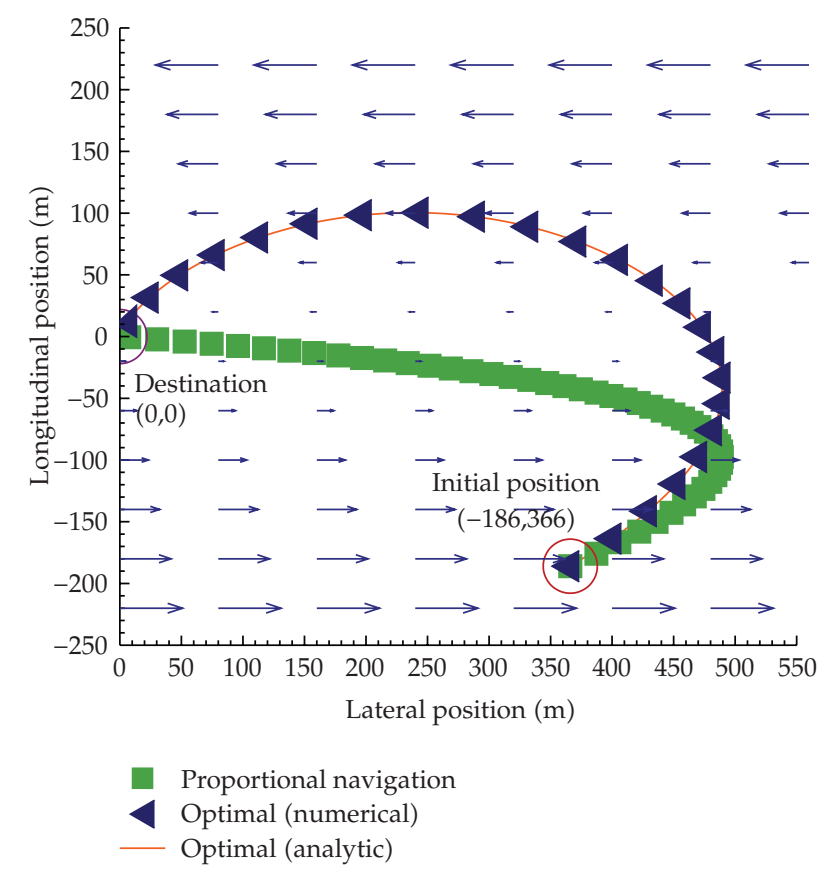

Figure 13: Navigation trajectories in a linear shearing current flow.

the vehicle under the guidance of $\mathrm{PN}$ is about to restart toward the destination. At the same instant, however, the optimal guidance has already made the vehicle arrive at the destination.

\subsection{QuasiOptimal Navigation}

The quasioptimal navigation is a fail-safe strategy introduced to react to failure in realizing the optimal navigation, due to environmental uncertainties or temporal malfunctions in sensors and actuators. The basic idea of the quasioptimal navigation is quite simple. The optimal heading reference during the quasioptimal navigation is revised repeatedly in response to the on-site request to prevent failure in on-going optimal navigation. According to Bellman's principle of optimality [25, 26], once we have failed in tracking the optimal trajectory, the best policy we can take from then on is to construct and follow a revised optimal trajectory starting at the present state. To derive the revised optimal trajectory, the optimal guidance law (5.1) has to be applied and solved again by using the proposed numerical solution procedure, that is, AREN, taking the present vehicle position as the new initial position. The whole trajectory obtained here is not optimal, since it already has included the past nonoptimal interval. Nonetheless, it is evidently the best trajectory we can take under the present situation, so that we call the corresponding navigation the quasioptimal navigation. Optimal and quasioptimal navigation trajectories in a time-varying current flow are shown in Figure 15.

The current distribution in this example is the same one that we took in the previous example. In this example, however, while the optimal navigation is performed with the exact information about the current flow distribution, assuming a situation of incorrect localization due to sensor failure, mismatched current flow information is fed to the vehicle guidance 


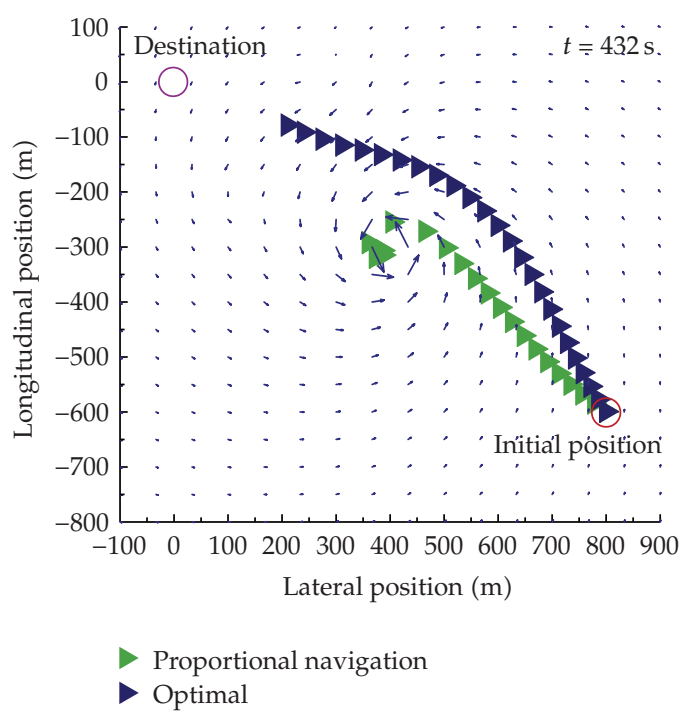

(a)

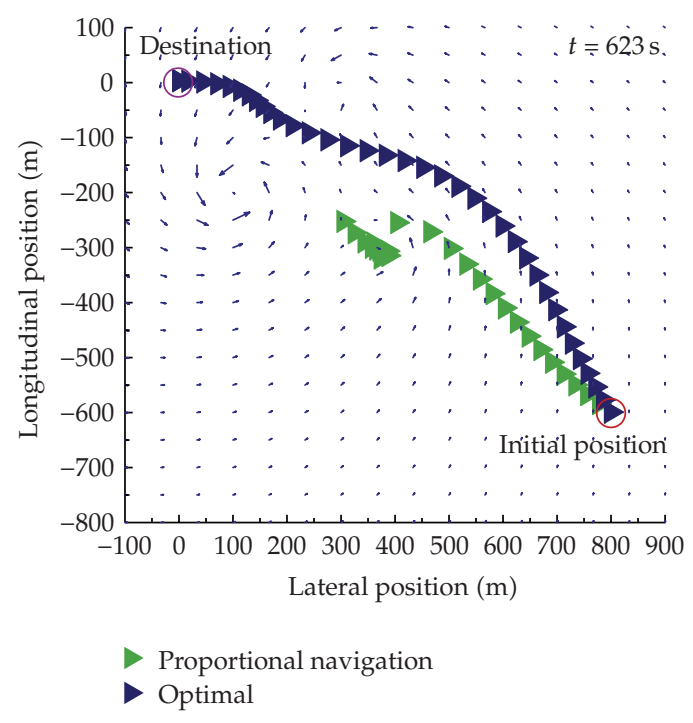

(b)

Figure 14: Navigation trajectories in a time-varying current flow at (a) $t=432 \mathrm{~s}$ and (b) $t=623 \mathrm{~s}$.

controller in the quasioptimal case. The time interval during which mismatched information is taken is $0.0 \sim 252.0 \mathrm{~s}$. Starting at $252.0 \mathrm{~s}$, optimal guidance revised on the basis of the correct current flow information achieves the quasioptimal navigation. Figure 16 shows the time sequence of the vehicle headings during the optimal and the quasioptimal navigations.

As expected naturally, the performance of the quasioptimal navigation is not as high as that of the optimal one. While the optimal guidance completes the navigation at $623 \mathrm{~s}$, the quasioptimal one continues it until 702 s. Note that in Figure 16, an abrupt heading change occurs at $252.0 \mathrm{~s}$ during the quasioptimal navigation.

\subsection{Optimal Navigation in Northwestern Pacific}

In what follows, we try to accomplish the minimum-time navigation within a real sea environment. The sea region selected for this optimal navigation example is located in the Northwestern Pacific Ocean near Japan. The current field considered here is an actual measurement of the surface flow provided by the Japan Meteorological Agency, available at http:/ / www.data.kishou.go.jp/db/kobe/db_kobe.html. The most notable environmental characteristic in this sea region is the current field dominated by the Kuroshio. The Kuroshio is a strong western boundary current in the Northwestern Pacific Ocean, flowing northeastward along the eastern coast of Japan [29]. As seen in Figure 18, Kuroshio-induced flows moving eastwards constitute the principal stream in this region.

In the current field data from the database of the Japan Meteorological Agency, current velocity is defined only on the predefined, large-scale grid nodes covering the sea region. As noticeable from (5.1), however, in order to derive the optimal heading reference, current velocity and its gradient at every vehicle position have to be available. As a remedy for this data deficiency, we estimate the current velocity and its gradient by interpolating the predefined values on grid nodes surrounding the present vehicle position. In applying 


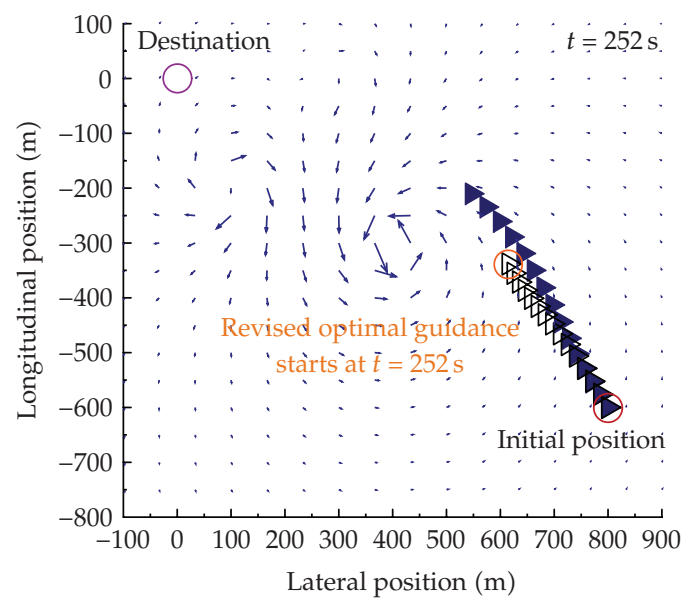

(a)

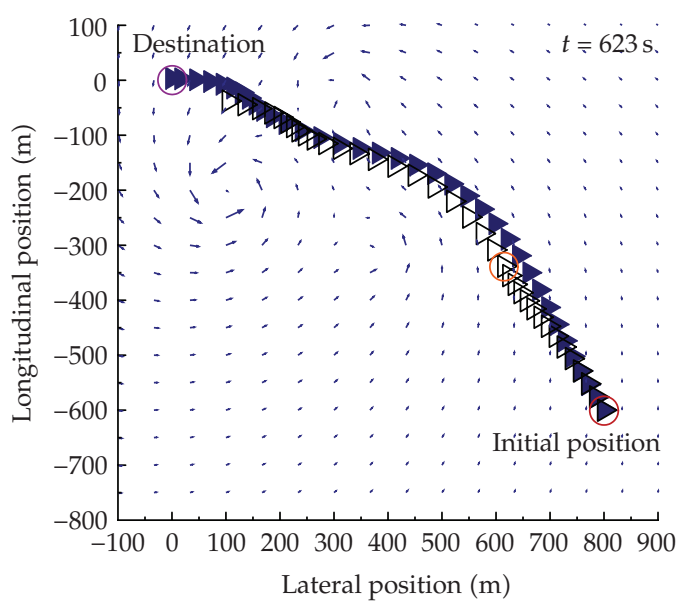

(b)

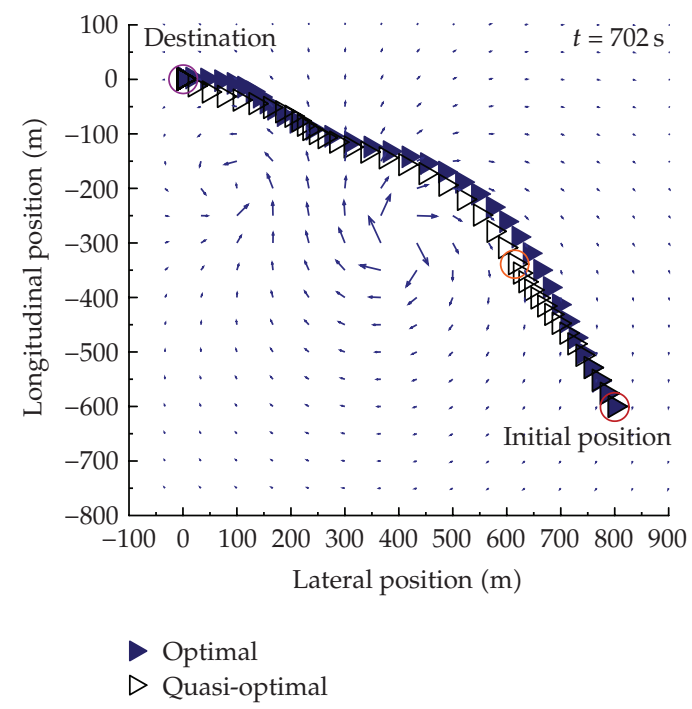

(c)

Figure 15: Navigation trajectories in a time-varying current flow at (a) $t=252 \mathrm{~s}$, (b) $t=623 \mathrm{~s}$, and (c) $t=$ $702 \mathrm{~s}$.

the interpolation, the nearest grid node to the present vehicle position has to be identified first. Then, the current velocity at the present vehicle position is estimated by 2D biquadratic interpolation utilizing values on the nearest node and surrounding eight nodes, as shown in Figure 17. Gradients of current velocities are obtained by the same manner. Since the velocity gradients are not provided from the database, however, prior to the interpolation, we calculate their nodal values by finite difference approximations.

The description of the navigation to be optimized here is as follows. Starting from an initial position, the vehicle is to transit to a destination in a mission-specified area, where an undersea survey mission is to be undertaken. Taking into account the inshore or harbor launch, the vehicle is made to start from the initial position off Minamiizu, the southern extreme of the Izu peninsula (Figure 18). 


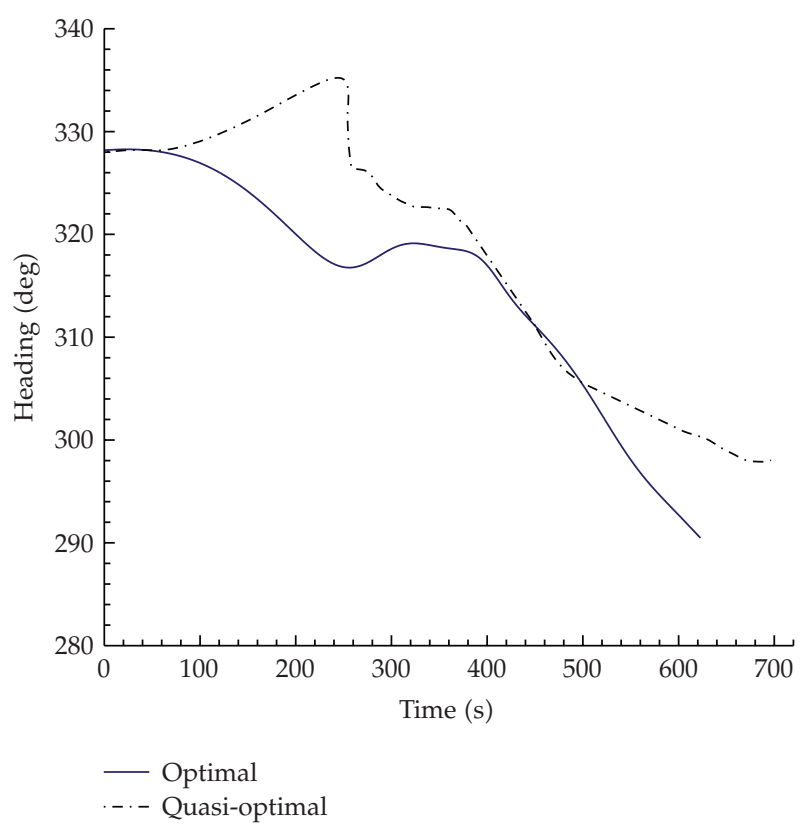

Figure 16: Time sequence of vehicle headings.

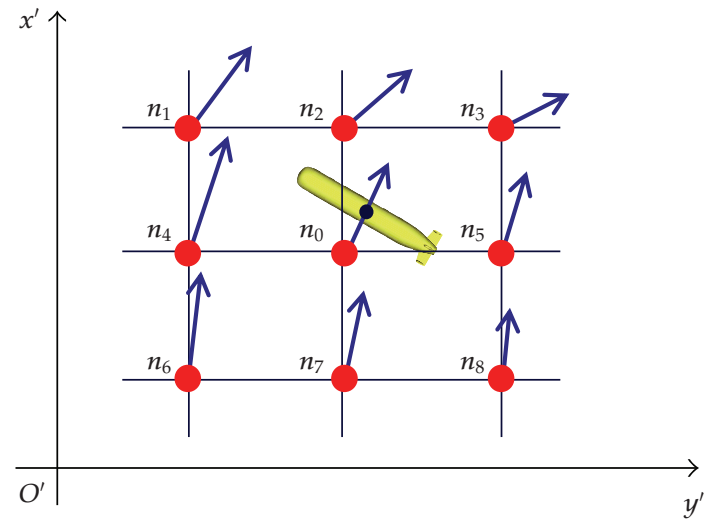

Figure 17: Cut sea area for interpolating current velocity and its gradient. When $n_{0}$ is the nearest grid node to the present vehicle position, current velocity and its gradient on that position are estimated using their values on $n_{0}$ to $n_{8}$.

Figure 18 shows the navigation trajectories achieved by the PN and optimal guidance. As shown in the figure, like the preceding examples in which exact values of current velocity and its gradients are available anywhere in the navigation region, the vehicle tracks the optimal reference trajectory with a negligibly small deviation. This indicates that our strategy of optimal navigation is also valid in the real sea current data, originally defined only on the coarsely defined discrete grid nodes.

In Figure 18, with the vehicle moving under PN, having reached the region of the mainstream of Kuroshio, its speed relative to the ground is remarkably reduced. This is 


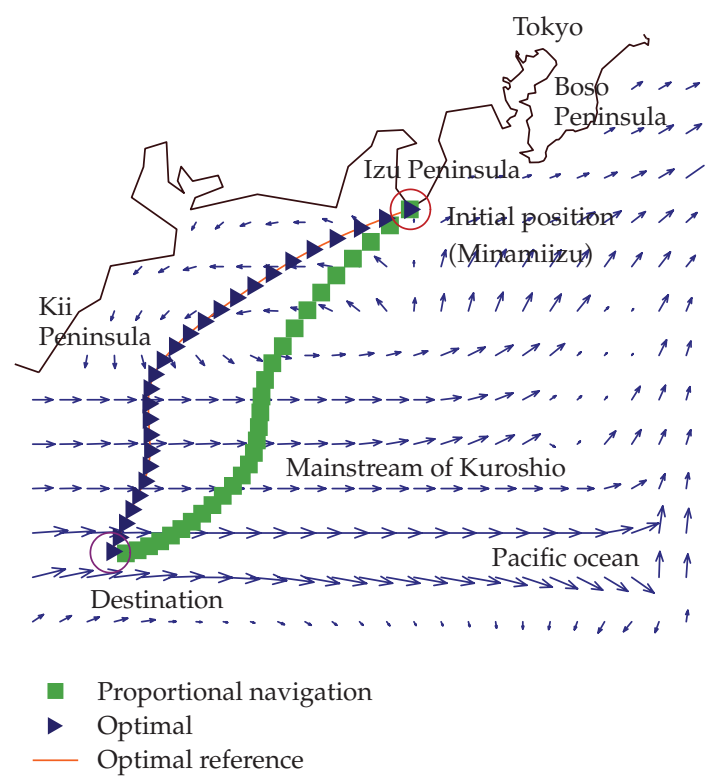

Figure 18: Navigation trajectories in a Northwestern Pacific Ocean region. The current data is built from actual measurements.

because in this region, for the vehicle following the guidance of $\mathrm{PN}$, the direction of its advance velocity is placed out-of-phase with the direction of the mainstream of Kuroshio. In the optimal navigation, the former segment of the navigation trajectory is formed along the shoreline until the vehicle reaches a point off the southern extreme of Kii peninsula. By taking this route, the vehicle attains a speed increase, riding the coastal current mainly flowing westwards. Note that upon the vehicle reaching a point off the southern extreme of the Kii peninsula, the optimal trajectory takes a large turn, slightly rolling back eastward from the destination. This slight rollback is the result of the optimal guidance's action to prevent the vehicle's advancing direction from being out-of-phase with that of the mainstream of Kuroshio, leading to the optimal navigation trajectory shown. The optimal trajectory obtained reveals one of the significant advantages of our approach over GA-based path planning which is not able to generate the optimal path with interim backward intervals by its nature $[7,9]$.

Navigation times by PN and optimal guidance are 232198 and 212006 s, respectively, indicating an $8.7 \%$ decrease in navigation time by the optimal guidance proposed.

\section{Conclusions and Future Works}

In this article, model-based analysis and synthesis to the following three research fields in AUV design and development have been presented.

(i) Dynamic system modelling of an AUV.

(ii) Motion control design and tracking control application.

(iii) Optimal guidance of an AUV in environmental disturbances. 
In the dynamic system modelling of the AUV R-One, we evaluated the hydrodynamic loads by using CFD analyses. Then, by differentiating a hydrodynamic load with respect to the amount of a perturbation, corresponding stability derivatives were obtained. Using the stability derivatives evaluated, we built up the dynamic model of the R-One, which is characterized to be 6-DOF [3 longitudinal (surge, heave, pitch) + 3 lateral (sway, roll, yaw)], linear, and multiple-input multiple-output (MIMO).

Depth and heading control systems are designed by employing controller models based on the PID compensations. In the PID-tuning, model-based simulations for the depth and the heading controls are exploited in determining the optimal gains.

Concerning the guidance problem of AUVs moving in sea environmental disturbances, a newly developed procedure for obtaining the numerical solution of the optimal guidance law to achieve the minimum-time navigation has been presented. The optimal heading is obtained as the solution of the optimal guidance law, which is fed to the heading controller as the optimal reference. Reduced computational cost is one of the outstanding features of the proposed procedure. Numerical calculations of the optimal navigation examples presented in this article except for the last one are completed within 10 minutes on a single core $2.4 \mathrm{GHz}$ windows XP platform. Moreover, unlike other path-finding algorithms such as DP or GAs, our procedure does not require a computation time increase for the timevarying problems.

As a fail-safe strategy for putting the proposed optimal navigation into execution, the concept of quasioptimal guidance has been proposed. The fact that there actually are several possible actions lessening the chance of achieving optimality emphasizes the practical importance of the quasioptimal navigation.

We have not considered the problem of unknown or nondeterministic currents. Our approach cannot be applied to an entirely unknown environment. For a sea region containing partially or coarsely defined currents, however, an estimated distribution can be built via interpolation and extrapolation, as shown in the last navigation example. The estimation possibly contains more or less uncertainty. Notably, however, it is the quasioptimal strategy that can cope with the environmental uncertainty. When the uncertainty in the estimation is significant, convergence may not be guaranteed.

\section{Acknowledgments}

The first author would like to express special thanks to Dr. Makio Kashino, Dr. Eisaku Maeda, and Dr. Yoshinobu Tonomura with NTT.

\section{References}

[1] B. Etkin, Dynamics of Flight_-Stability and Control, John Wiley \& Sons, New York, NY, USA, 1996.

[2] D. McRuer, I. Ashkenas, and D. Graham, Aircraft Dynamics and Automatic Control, Princeton University Press, Princeton, NJ, USA, 1990.

[3] E. V. Lewis, Ed., Principles of Naval Architecture. Vol III. Motions in Waves and Controllability, The Society of Naval Architects and Marine Engineers, Jersey City, NJ, USA, 1989.

[4] M. A. Park, L. L. Green, R. C. Montgomery, and L. L. Raney, “Determination of stability and control derivatives using computational fluid dynamics and automatic differentiation," in Proceedings of the 17th AIAA Applied Aerodynamics Conference, Norfolk, Va, USA, June 1999, AIAA paper 99-3136.

[5] F. T. Johnson, E. N. Tinoco, and N. J. Yu, "Thirty years of development and application of CFD at Boeing commercial airplanes, Seattle," Computers \& Fluids, vol. 34, no. 10, pp. 1115-1151, 2005.

[6] T. Ohmori, "Finite-volume simulation of flows about a ship in maneuvering motion," Journal of Marine Science and Technology, vol. 3, no. 2, pp. 82-93, 1998. 
[7] A. Alvarez, A. Caiti, and R. Onken, "Evolutionary path planning for autonomous underwater vehicle in a variable ocean," IEEE Journal of Oceanic Engineering, vol. 29, no. 2, pp. 418-429, 2004.

[8] N. A. Papadakis and A. N. Perakis, "Deterministic minimal time vessel routing," Operations Research, vol. 38, no. 3, pp. 426-438, 1990.

[9] C. Hocaolglu and A. C. Sanderson, "Planning multi-paths using speciation in generic algorithms," in Proceedings of IEEE International Conference on Evolutionary Computation (ICEC '97), pp. 378-383, Indianapolis, Ind, USA, April 1997.

[10] D. A. Smallwood and L. L. Whitcomb, "Model-based dynamic positioning of underwater robotic vehicles: theory and experiment," IEEE Journal of Oceanic Engineering, vol. 29, no. 1, pp. 169-186, 2004.

[11] K. Kim and T. Ura, "Optimal and quasi-optimal navigations of an AUV in current disturbances," in Proceedings of IEEE/RSJ International Conference on Intelligent Robots and Systems (IROS '08), pp. 36613667, Nice, France, September 2008.

[12] T. Ura, "Development of autonomous underwater vehicles in Japan," Advanced Robotics, vol. 16, no. 1, pp. 3-15, 2002.

[13] CD-adapco, STAR-CD V3.2 User Guide Manuals, CD-adapco, New York, NY, USA, 2002.

[14] G. Iaccarino, "Predictions of a turbulent separated flow using commercial CFD codes," Journal of Fluids Engineering, vol. 123, no. 4, pp. 819-828, 2001.

[15] M. Garbey and C. Picard, "A code-independent technique for computational verification of fluid mechanics and heat transfer problems," Acta Mechanica Sinica, vol. 24, no. 4, pp. 387-397, 2008.

[16] J. L. Lumley, "Computational modeling of turbulent flows," Advances in Applied Mechanics, vol. 18, pp. 123-176, 1978.

[17] J. F. Thompson, "A composite grid generation code for general 3-D regions—the Eagle code," AIAA Journal, vol. 26, no. 3, pp. 271-272, 1988.

[18] F. M. White, Fluid Mechanics, McGraw-Hill, New York, NY, USA, 1988.

[19] T. Ura, T. Obara, S. Takagawa, and T. Gamo, "Exploration of Teisi Knoll by autonomous underwater vehicle "R-One Robot"," in Proceedings of MTS/IEEE OCEANS, vol. 1, pp. 456-461, Honolulu, Hawaii, USA, November 2001.

[20] T. Takagi and M. Sugeno, "Fuzzy identifications of systems and its application to modelling and control," IEEE Transactions on Systems Man and Cybernetics, vol. 15, no. 1, pp. 116-132, 1985.

[21] K. S. Narendra and K. Parathasarathy, "Identification and control of dynamic system using neural network," IEEE Transactions on Neural Network, vol. 1, no. 1, pp. 4-27, 1990.

[22] S. Haykin, Neural Networks, Prentice Hall, Upper Saddle River, NJ, USA, 1999.

[23] H.-X. Li and S. Guan, "Hybrid intelligent control strategy," IEEE Control Systems Magazine, vol. 21, no. 3, pp. 36-48, 2001.

[24] The Mathworks Inc., Control System Toolbox User's Guide, The Mathworks Inc., Natick, Mass, USA, 2007.

[25] F. L. Lewis and V. L. Syrmos, Optimal Control, A Wiley-Interscience Publication, John Wiley \& Sons, New York, NY, USA, 1986.

[26] A. E. Bryson and Y. C. Ho, Applied Optimal Control, Taylor \& Francis, Levittown, NY, USA, 1975.

[27] L. G. Crespo and J. Q. Sun, "Optimal control of target tracking with state constraints via cell mapping," Journal of Guidance, Control, and Dynamics, vol. 24, no. 5, pp. 1029-1031, 2001.

[28] W. H. Press, S. A. Teukolsky, W. T. Vetterling, and B. P. Flannery, Numerical Recipes in Fortran, Cambridge University Press, Cambridge, UK, 2nd edition, 1992.

[29] S. Imawaki, H. Ichikawa, M. Ikeda, A. Isobe, and M. Kamachi, "Introduction to special section: Kuroshio observation, state estimation and prediction," Journal of Oceanography, vol. 60, no. 3, pp. 265-268, 2004. 


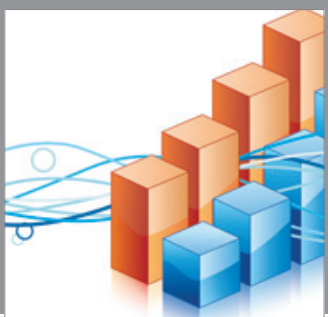

Advances in

Operations Research

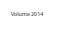

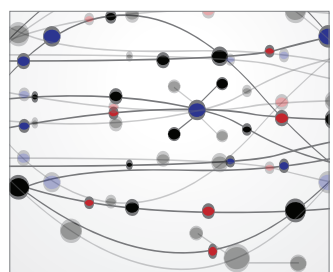

\section{The Scientific} World Journal
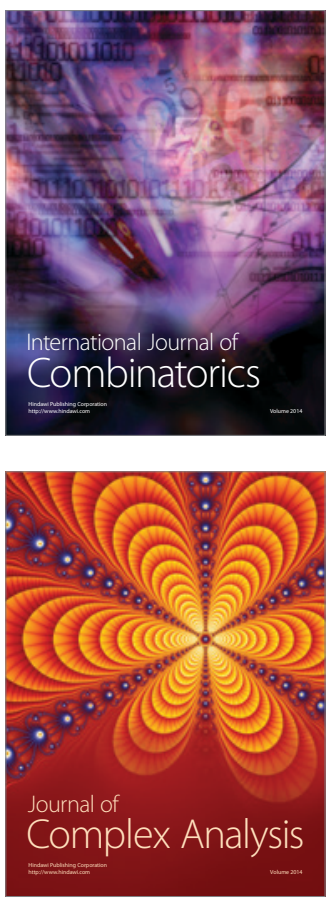

International Journal of

Mathematics and

Mathematical

Sciences
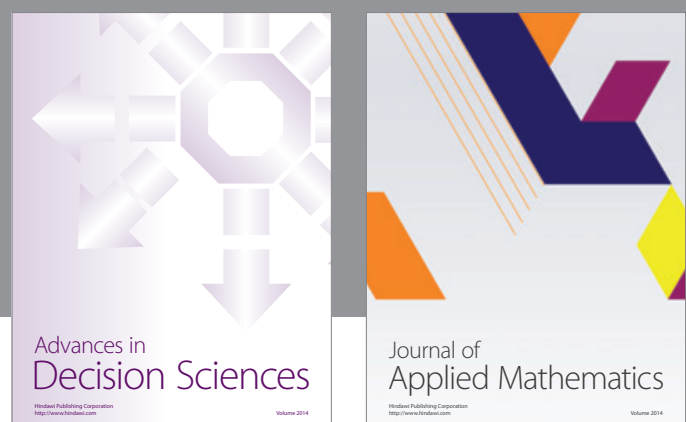

Journal of

Applied Mathematics
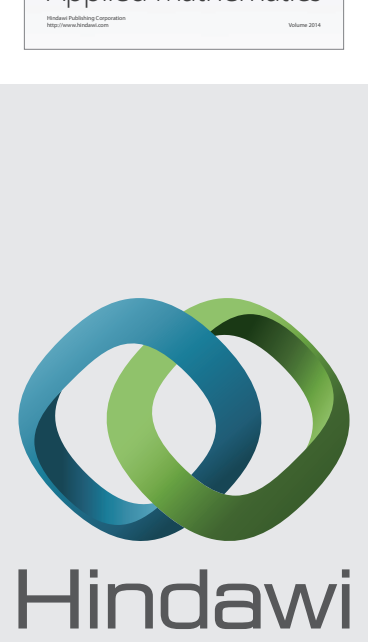

Submit your manuscripts at http://www.hindawi.com
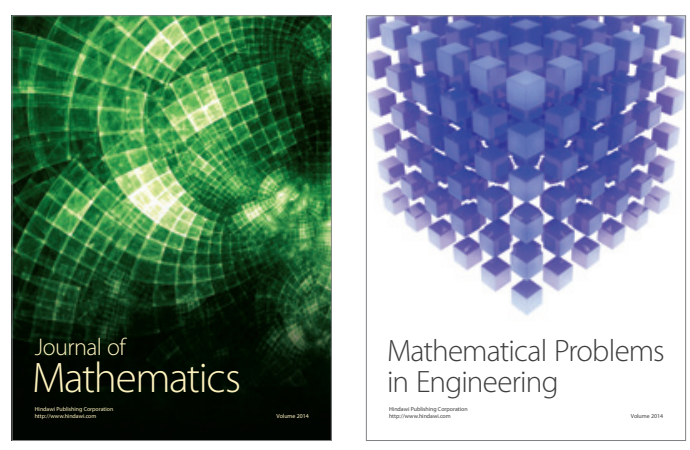

Mathematical Problems in Engineering
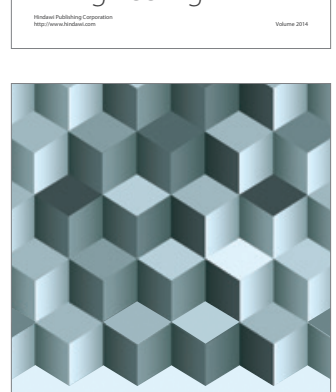

Journal of

Function Spaces
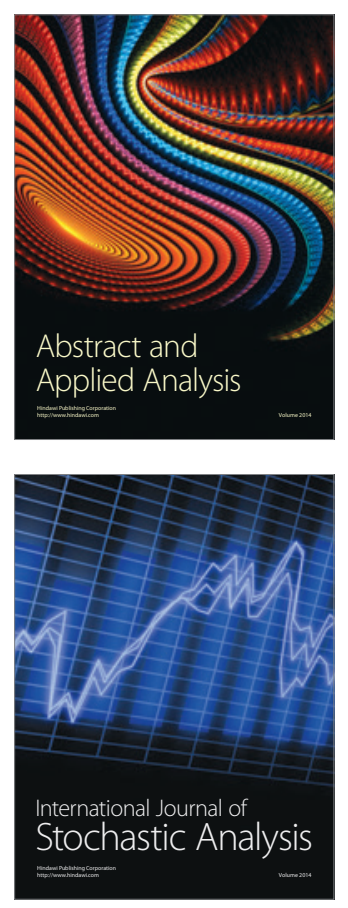

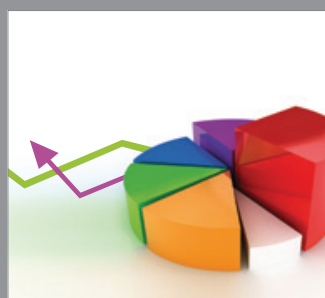

ournal of

Probability and Statistics

Promensencen
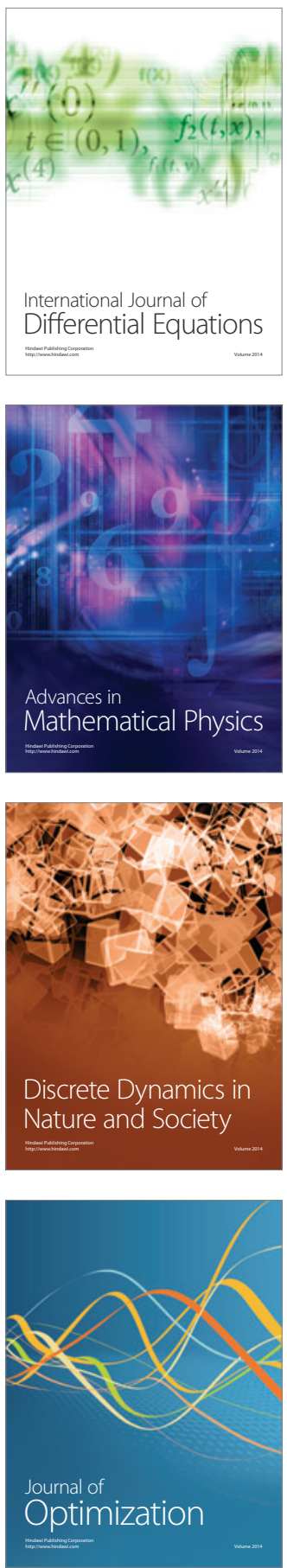